\title{
A global perspective for non-conservative dynamics
}

\section{Une perspective globale pour la dynamique non-conservative}

\author{
J. Palis ${ }^{1}$ \\ Instituto Nacional de Matemática Pura e Aplicada, Est. Dona Castorina 110, 22460-320, Jardim Botânico, RJ, Brazil
}

Received 19 January 2005; accepted 28 January 2005

Available online 27 April 2005

\begin{abstract}
Since Poincaré's fundamental work on the qualitative study of differential equations in 1881, the viewpoint of pursuing the description of the long range behavior of trajectories for "most" systems has been somewhat present in dynamics. About eight decades later, Smale was aiming exactly at that purpose when he introduced in the early sixties the concept of hyperbolic systems, which turns out to correspond to a large class of robust systems that gave rise to a basic theory of modern dynamics. Yet, hyperbolic systems are not typical since not every system can be approximated by a hyperbolic one, as can be seen by the famous example of the butterfly attractor provided by Lorenz, which is not hyperbolic and still robust or totally persistent under small perturbations of the initial flow. A string of other counter-examples were constructed in the late sixties and early seventies, and is to be noticed that all of them are related to cycles in dynamics, introduced by Poincaré, which will be focused here.

In the present paper, we wish to provide some perspective about this major question, proposing in qualitative terms what could be the main characteristics of a typical system. Indeed, we shall discuss partial successes and a possible strategy for proving a global conjecture on the finitude of large basin attractors and their stochastic stability for non-conservative dynamics; i.e. $C^{r}$ flows, diffeomorphisms, and transformations of compact, boundaryless manifolds or the interval, $r \geqslant 1$. We shall also impose the union of the attracting basins to have total probability in the ambient space (phase space). Thus, the aim of our conjecture is a description in a rather simple conceptual way of the long range behavior of a typical (positive) trajectory of a typical dynamical system: each trajectory has only finitely many choices (of attractors) where to accumulate upon in the future. We discuss some recent related results, including homoclinic bifurcations, dynamical robustness and persistence, as well as systems with a dominated or partially hyperbolic decomposition.

My perspective is that we should expect, no so long in the future, substantial progress about the main and some of the other conjectures in this paper in the $C^{1}$ topology context and for one-dimensional dynamics, as conveyed in the introduction. A key difficulty in higher dimensions and the $C^{r}$ topology, $r>1$, arises from the question whether densely, in the space of nonconservative dynamics, the systems display a dense subset of periodic orbits in their limit or nonwandering set. The question
\end{abstract}

E-mail address: jpalis@impa.br (J. Palis).

1 Partially supported by CNPq-Brazil and FAPERJ. 
has been positively settled only in the $C^{1}$ topology, a result due to Pugh in the sixties, and it remains open otherwise except for dimension one or flows on orientable surfaces. Still, in my view, the $C^{1}$ case is already iluminating of the darker realm of dynamics. Above all, the idea of having most systems with only finitely many attractors toward which almost all orbits are attracted to, seems to me a tempting one to be considered even in special and yet relevant settings.

(C) 2005 L'Association Publications de l'Institut Henri Poincaré. Published by Elsevier B.V. All rights reserved

\section{Résumé}

Depuis le travail fondamental de Poincaré sur l'étude qualitative des équations différentielles en 1881, l'idée de chercher la description du comportement à long terme des trajectoires pour «la plupart» des systèmes a été présente d'une certaine façon en dynamique. Environ huit décennies plus tard, Smale s'attelait exactement à cette tâche quand il introduisit, au début des années soixante, le concept de systèmes hyperboliques, qui correspondent finalement à une grande classe de systèmes robustes qui donnèrent naissance à une théorie de base de la dynamique moderne. Cependant, les systèmes hyperboliques ne sont pas typiques puisque n'importe quel système ne peut pas être approché par un système hyperbolique, comme le montre le célèbre attracteur «papillon» découvert par Lorenz, qui n'est pas hyperbolique mais toujours robuste ou totalement persistant par des petites perturbations du flot initial. Une série d'autres contre-exemples furent construits à la fin des années soixante et au début des années soixante-dix, et l'on doit remarquer que tous sont liés à des cycles en dynamique, introduits par Poincaré, et que nous allons considérer ici.

Dans cet article, nous souhaitons donner une vision de cette question majeure, proposer en des termes qualitatifs ce que pourraient être les principales caractéristiques d'un système typique. En effet, nous discuterons des succès partiels et d'une possible stratégie pour prouver une conjecture globale sur la finitude des attracteurs des grands bassins et leur stabilité stochastique pour les dynamiques non-conservatives, à savoir, les flots, les difféomorphismes et les transformations des variétés compactes sans bords ou de l'intervalle de classe $C^{r}$ pour $r \geqslant 1$. Nous imposerons aussi que l'union des bassins attracteurs soit de probabilité un dans l'espace ambiant (l'espace des phases). Ainsi, le but de notre conjecture est une description, d'une manière relativement simple conceptuellement, du comportement à long terme d'une trajectoire (positive) typique d'un système dynamique typique : chaque trajectoire n'a qu'un nombre fini de choix (d'attracteurs) où s'accumuler dans le futur.

Nous discuterons de quelques résultats connexes récents, notamment des bifurcations homocliniques, de la robustesse dynamique et de la persistance, de même que des systèmes avec une décomposition dominée ou partiellement hyperbolique.

Mon point de vue est que nous devrions connaître à moyen terme des progrès substanciels à propos de la conjecture principale et d'autres conjectures de cet article dans le contexte de la topologie $C^{1}$ et pour la dynamique de dimension un, comme cela a été expliqué dans l'introduction. Une difficulté majeure en dimension supérieure et en topologie $C^{r}$ pour $r>1$ provient de la question de savoir si, de façon dense dans l'espace des dynamiques non-conservatives, les systèmes possèdent un sous-ensemble dense d'orbites périodiques dans leur ensemble limite ou leur ensemble «non-récurrent». La question a été résolue seulement dans le cas $C^{1}$ : c'est un résultat de Pugh dans les années soixante; elle reste ouverte dans les autres cas, sauf en dimension un ou pour le flot sur des surfaces orientables. De mon point de vue, même le cas $C^{1}$ est déjà éclairant dans le domaine inconnu de la dynamique. Avant tout, l'idée d'avoir souvent des systèmes avec un nombre fini d'attracteurs vers lesquels presque toutes les orbites sont également attirées me semble être une idée attrayante à considérer même dans des contextes particuliers mais pourtant pertinents.

(C) 2005 L'Association Publications de l'Institut Henri Poincaré. Published by Elsevier B.V. All rights reserved

\section{Introduction}

We shall present in this paper a global scenario for dissipative or, more precisely, non-conservative dynamics, i.e. $C^{r}$ flows, diffeomorphisms and transformations, $r \geqslant 1$, on compact boundaryless manifolds or intervals on the real line. The main focus concerns a conjecture on the denseness, in the $C^{r}$ topology, of systems having only finitely many attractors, the attractors being sensitive to initial conditions (chaotic) or just periodic sinks and the union of their basins of attraction having total Lebesgue probability. The attractors should also be stochastically stable in their basins of attraction with respect to random perturbations in a finite dimensional space of parameters. The conjecture is formally presented and discussed in Section 2. In Sections 2 and 3, other related conjectures are presented as well as a number of recent partial results. From them we have inferred a possible strategy to prove 
that our global scenario may indeed be valid. Basic definitions and some known or motivating results are presented in Section 2.

Our conjecture corresponds to a probabilistic and subtle version of another main one proposed by Smale in the sixties, but by the end of that decade many counter-examples to it had been provided, including by Smale himself. This fact certainly led for quite a while to a vacuum with respect to the possibility of formulating a global scenario for dynamics, through which we could provide key properties of a typical dynamical system. Smale's proposal stated that systems with a hyperbolic structure, form an open and dense subset of all systems in the $C^{r}$ topology, $r \geqslant 1$ (see Section 2).

Most remarkably, towards the end of the sixties Kolmogorov when visiting IMPA, Rio de Janeiro, stated to colleagues there that the global study of dynamical systems could not go very far without the use of new additional mathematical tools, like probabilistic ones. Of course, the work of Sinai, Arnold and Anosov, among others, had already such a flavor at that point. But, I interpreted Kolmogorov's view, as expressed to me by Elon Lima sometime afterwards, as referring to Smale's proposal of a global scenario for dynamics, since counter-examples to the denseness of hyperbolic systems were already known. For years to come, I kept such a remarkable insight in my mind.

So, I take the occasion to pay Kolmogorov and Smale my tribute.

The collapse of Smale's conjecture excluded the case of flows on disks and surfaces, as well as for onedimensional dynamics. Indeed, new excellent results have just been announced proving it in the real onedimensional case, as shall be discussed in Section 2.5.

On the other hand, for one-dimensional real or complex dynamics, the conjecture presented here goes even further than indicated in the Abstract:

- For almost all parameter values, the corresponding dynamical systems display finitely many attractors which are periodic sinks or carry an absolutely continuous invariant probability measure.

Concerning the main conjecture, there is the following string of outstanding recent results in the real onedimensional case, that culminates with its full solution for unimodal maps, i.e., maps with only one critical point.

A first breakthrough is due to Lyubich [71], using results by Martens and Nowicki [74] and previously Sullivan [115] and McMullen [76], among others. To state his result, we consider the quadratic family

$$
f_{x}(x)=\operatorname{ax}(1-x), \quad f_{a}:[0,1] \rightarrow[0,1], \quad 0<a \leqslant 4,
$$

that plays a key role in the theory of dynamics of interval maps. We then have

Lyubich [71]: For almost every $a$ in $(0,4], f_{a}$ either has a hyperbolic attractor (sink) attracting almost all orbits or else it is chaotic, i.e., it has an absolute continuous invariant probability measure, which is unique and ergodic (and so a SRB measure).

Following that, there was an intense activity with other authors joining in the efforts to successfully achieve a definite picture of the typical dynamics for unimodal maps.

Avila, de Melo and Lyubich [17]: Same conclusion as above in the analytic case, assuming negative Schwarz derivative and making use of a key structure (laminations corresponding to the topological conjugacy classes) in the space of infinitely renormalizable maps.

Avila and Moreira: The main conjecture is true for non-degenerate analytic families, in particular the above quadratic one [20], and even just generic $C^{k}$ families, $k \geqslant 2$, of unimodal maps [18,21]. Moreover, surprisingly, there exists an explicit formula that gives the eigenvalues of periodic orbits as a function of corresponding kneading sequences and of the kneading sequence of the critical orbit, valid for almost all parameters in any generic analytic family of unimodal maps [19]. They also announced that the main conjecture holds in the complement of a set of positive codimension.

In my perspective these last results shall soon be fully extended to the multimodal case. 
As mentioned above, we shall discuss in Section 3 other relevant conjectures and questions, particularly concerning homoclinic bifurcations and robustness of transitive sets. Motivated by such questions and some of the recent results presented here, we have set up a possible strategy to prove the validity of the global scenario for non-conservative dynamics as proposed in 1995 in a meeting in honor of A. Douady [90].

I wish to thank W. de Melo, C.G. Moreira and specially to M. Viana for their precious comments and information while I was preparing this text. I also want to mention that Viana's book with Bonatti and Diaz [28] has a broad account of present day dynamics, being more comprehensive in the non-conservative case.

\section{Basic definitions and results: the global conjecture on the finitude of attractors}

In this section we shall discuss the basic ingredients leading to our main conjecture. We also discuss Smale's global conjecture in the sixties proposing the class of hyperbolic (or structurally stable) systems as paradigm for typical dynamics.

\subsection{Sinai-Ruelle-Bowen probability measure}

This remarkable notion was introduced by Sinai [112] and proven by him to exist for Anosov or globally hyperbolic diffeomorphisms, as defined in Section 2.4 below. Subsequently, it was shown to exist by Ruelle [107] and Ruelle and Bowen [32] for hyperbolic diffeomorphisms and flows.

The definition is expressed in rather simple terms, but its content is quite meaningful in describing the dynamics of attractors when it's possible to show that they carry SRB-measures, which is usually a hard task.

Let $A$ be an attractor for $f$, i.e. there is a set of points in the phase space with positive Lebesgue probability whose future orbits tend to $A$, as the number of iterates tends to infinite. The set of orbits attracted to $A$ in the future is called its basin. The definition for flows is entirely similar. Let $\mu$ be an $f$-invariant probability measure on $A$. Then $\mu$ is called a SRB (Sinai-Ruelle-Bowen) measure for $(f, A)$ if we have for any continuous map $g$, that

$$
\lim _{n \rightarrow \infty} \frac{1}{n} \sum g\left(f^{i}(x)\right)=\int g \mathrm{~d} \mu
$$

$x \in E \subset B(A)$ basin of attraction for $A$, with $m(E)>0$, where $m$ denotes Lebesgue measure. It's common to have $E$ with total probability in $B(A)$ and so, in such a case, the above convergence holds for a typical trajectory attracted to $A$ in the future.

\subsection{Stochastic stability}

For attractors carrying SRB-measures, we can investigate the question of their stability or persistence when we slightly perturb the dynamics, more particularly from a probabilistic viewpoint. Thus, we shall follow Kolmogorov's perspective. Indeed, the more classical concept of structural stability, introduced by Andronov and Pontryagin in the 30's, remarkably turned out to be too close or even equivalent to hyperbolicity (see Section 2.5). However, there are open sets of structurally unstable systems, and so we consider instead the concept of stochastic stability.

Let $A$ be an attractor for $f, \mu$ be a SRB invariant probability for $(f, A)$ and $f$ be an element of a finite dimensional parameter-space of $C^{r}$ maps, $r \geqslant 1$. Random Lebesgue choice of parameters gives rise to maps $f_{j}$, $j=0,1, \ldots$, that are $\varepsilon$-near $f$ in parameter space, some $\varepsilon>0$. Let $z_{j}=f_{j} \circ \cdots \circ f_{1}\left(z_{0}\right), z_{0} \in B(A)$.

We call $(f, A, \mu)$ stochastically stable if given a neighborhood $V$ of $\mu$ in the weak topology, the weak limit of $\frac{1}{n} \sum_{j=0}^{n-1} \delta_{z_{j}}$ is in $V$ for a.a. $\left(z_{0}, f_{1}, f_{2}, \ldots\right)$ if $\varepsilon$ is small, where $\delta_{z_{j}}$ stands for the Dirac measure at $z_{j}$.

Variants of this definition, including the case of flows, are briefly presented in [90] and references provided; see also [28] for a comprehensive discussion. 


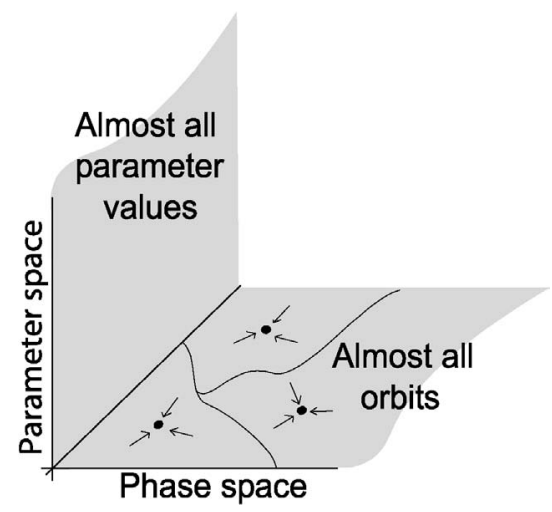

Fig. 1.

\subsection{Attractors}

As defined in Subsection 2.1 on SRB-measures above, attractors should be the positive limit set for the orbits starting at a subset of the phase space (space of events) with positive Lebesgue probability. Often, they attract the orbits of a full measure set in a neighborhood of them. We add to the definition of attractor that it must be transitive, i.e., it must display a dense orbit, so it cannot be decomposed into strictly smaller attractors. The simplest example for a diffeomorphism $f$ is that of an attracting periodic orbit: the eigenvalues of $\mathrm{d} f^{n}(p)$ have norm less than one, where $p$ is the periodic orbit and $n$ its period. Another well known case is that of the whole torus $T^{2}$ for the map induced by the linear one $L$ on $\mathbb{R}^{2}$ defined by $L(1,0)=(2,1), L(0,1)=(1,1)$.

Attractors are of paramount importance in dynamics since they mold the future behavior of many orbits, i.e. the ones in their basin of attraction. What we want mainly to discuss in the present paper is our conjecture that a finite number of attractors should in fact attract Lebesgue almost all future orbits, at least for a dense subset of dynamical systems in the $C^{r}$ topology, $r \geqslant 1$. We can go even further when considering finite dimensional parameter families of dynamics, by requiring that such a property holds for dynamical systems corresponding to Lebesgue almost all parameter values. The precise statement of the conjecture is at the end of the present section.

We want to emphasize that we shall play, in probabilistic terms, with almost all orbits in the phase space (space of events) of dynamical systems corresponding to almost all parameter values, as suggested by Fig. 1 .

So, figuratively what we wish to propose is that "small accidents" both in terms of Lebesgue measure in the parameter space of systems as well as in the phase space are not "very relevant" in order to provide such a global scenario for dynamics.

We now give the definition of a hyperbolic system and, in particular, of a hyperbolic attractor, and then briefly present important examples of both hyperbolic and non-hyperbolic attractors. They all shall have at least some degree of persistence. Formally, they shall exist for a positive probability set of parameter values. Or, sometimes even more sharply, for an open set in the space of parameters, in which case the attractors are called robust. The hyperbolic attractors are robust and, historically, the only ones known to be robust until the appearance of Lorenzlike attractors, as discussed in Section 2.6.

\subsection{Hyperbolicity}

A diffeomorphism $f: M \rightarrow M$ is called hyperbolic if on its limit set $L$, that is the closure of the sets where the orbits accumulate in the future or in the past, we have a decomposition of the tangent bundle of $M$ at $L$, $T_{L} M=E^{s} \oplus E^{u}$, such that $\mathrm{d} f\left|E^{s}, \mathrm{~d} f^{-1}\right| E^{u}$ are uniform contractions. 
In the case of a flow $X_{t}, t \in \mathbb{R}$, generated by a vector field $X$, we require that

$$
T_{L} M=E^{s} \oplus E^{0} \oplus E^{u} .
$$

Here, $E^{0}$ is one-dimensional and tangent to $X$, outside its singularities (a finite number) and

$$
\begin{aligned}
& \left\|\mathrm{d} X_{t}\left|E^{s}\|,\| \mathrm{d} X_{-t}\right| E^{u}\right\| \leqslant C \mathrm{e}^{\lambda t}, \quad t \in \mathbb{R}, \\
& C>0, \quad 0<\lambda<1 .
\end{aligned}
$$

In the cases where such bundle decompositions occur in the whole ambient manifold, we refer to such globally hyperbolic diffeomorphisms or flows as Anosov ones. Recall that associated to the orbits of a hyperbolic set there are stable and unstable manifolds, corresponding to the contracting and expanding sub-bundles by the action of the derivative of the dynamical system. Often we refer to a hyperbolic system as satisfying Axiom $A$ or more precisely when its non-wandering set, which may be larger than the limit set, is hyperbolic. For our discussion here, such a difference is not very relevant. Sometimes, by abus de langage, we refer to hyperbolic systems as having hyperbolic limit sets and all stable and unstable manifolds transversal. They form a robust set of dynamics, i.e., an open set in every $C^{r}$ topology, $r \geqslant 1$. Also, sometimes we refer to an Axiom A system as being hyperbolic and without cycles among the different closed, invariant and transitive subsets, in which the (hyperbolic) limit set can be decomposed. They, again, form a robust set of dynamics [95,114].

A very notable result in dynamics states in a simplified form (see additional condition below concerning invariant manifolds) that

$$
\text { hyperbolicity } \stackrel{C^{1}}{\sim} \text { structural stability }
$$

We recall that a diffeomorphism $f$ is $C^{r}$ structurally stable, or just dynamically stable or just stable, if for any $C^{r}$ small perturbation $g$ of $f$, there is a homeomorphism $h$ of the phase space such that $h f(x)=g h(x)$, for all points $x$ in the phase space. For flows, we require the existence of a homeomorphism $h$ sending trajectories of the initial flow to the trajectories of any small $C^{r}$ perturbation.

A beautiful page of dynamics is the solution, in the $C^{1}$ topology, of the so called Stability Conjecture, stating that a system is $C^{r}$ structurally stable if and only if it is hyperbolic and all the stable and unstable manifolds associated to the orbits in the limit set are in general position, i.e., transversal. In the equivalence indicated above, we didn't mention explicitly the transversality condition on stable and unstable manifolds because it corresponds to the easiest part of the conjecture.

That stability holds for hyperbolic systems has been proved, in various settings, by Anosov [10], Palis [89], Palis and Smale [91], Robbin [103], de Melo [39], Robinson [104] in the sixties and early seventies. The converse was completed in the eighties by Mañé [73] for diffeomorphisms and in the nineties by Hayashi [51] for flows, after earlier contributions by Liao [66,67], Sannami [109] and Mañé [72].

Predecessors to such works are the pioneering ones by Andronov and Pontryagin for flows on disks and later on Peixoto for flows on orientable surfaces, as cited in the next subsection.

\subsection{Conjecture (Smale)}

Smale's conjecture concerning a typical dynamical system formulated in the sixties, can simply be stated as follows: Every system can be approximated by a hyperbolic one. If the conjecture was true, the hyperbolic systems by denseness and robustness would be typical of dynamics.

A few years afterwards counter examples abounded, due to Smale [114], Abraham and Smale [1], Simon [111] and others. More strikingly, Newhouse [86] provided counter-examples for $C^{2}$ surface diffeomorphisms using arithmetic difference of "thick" Cantor sets (thickness is a kind of fractal dimension). He did so by unfolding homoclinic tangencies, as in Section 3.2, to generate open sets of nonhyperbolic diffeomorphisms containing dense subsets of maps exhibiting homoclinic tangencies. Newhourse's idea does not hold in the $C^{1}$ category, 
by Ures [119]. There were also the beautiful Lorenz butterfly attractor that came to our attention only in the early seventies and the Hénon attractor, both of which we shall much comment in the sequel.

In fact, Smale's conjecture was known for flows on disks [9] and orientable surfaces [99], and consequently for diffeomorphisms of the circle. Smale was initially inspired by these results that correspond to pioneering works by Andronov and Pontryagin in the thirties and Peixoto in late fifties.

More recently, it has been independently proved by Swiatek and Graczyk [49] and Lyubich [71] that the hyperbolic maps are dense among real quadratic ones. Subsequently, Kozlovski [59,60] extended this result to $C^{3}$ unimodal mappings. A remarkable recent theorem has been announced in two parts by Kozlovski, Shen and Van Strien [61,62], namely the density of the hyperbolic transformations among $C^{r}$ maps of the interval, for $r \geqslant 2$, the case of $r=1$ being also included by a much previous theorem of Jakobson [54]. The result of Kozlovski, Shen and Van Strien not only answers Smale's conjecture in one real dimension, but opens the way for proving the main conjecture in the present paper in full generality for maps of the interval or the circle.

It is important to observe that for the one-dimensional complex case, i.e., rational maps of the Riemman sphere, the question remains wide open.

\subsection{Beyond hyperbolic attractors}

For dynamics, the most interesting examples of attractors are the ones that are robust under small perturbations of the dynamics, as in the hyperbolic and Lorenz-like cases, or which are at least probability persistent (i.e., the attractor exists for a positive probability in parameter space), as in the Hénon-like case.

As mentioned before, the simplest attractors are the ones consisting of a simple fixed or periodic hyperbolic orbit. In the hyperbolic case, the other extreme corresponds to attractors consisting of the whole ambient manifold, like the one induced by hyperbolic toral automorphisms. This example turns out to be a particular case of Anosov diffeomorphisms for which we assume (uniform) hyperbolicity at all points of the manifold [10]. In this vein, it is still an open question whether it is true for all Anosov diffeomorphisms that the whole manifold consists of a (transitive) attractor. This is generally not true for Anosov flows already in dimension three [46].

The remarkable Lorenz "butterfly" attractor, whose original equations are in Fig. 2, was the first known case of a fully persistent or robust (it exists for all sufficiently small variations of the initial parameter values) and (transitive) attractor which is not hyperbolic [70]. Notice that its singularity $(0,0,0)$ is accumulated by hyperbolic

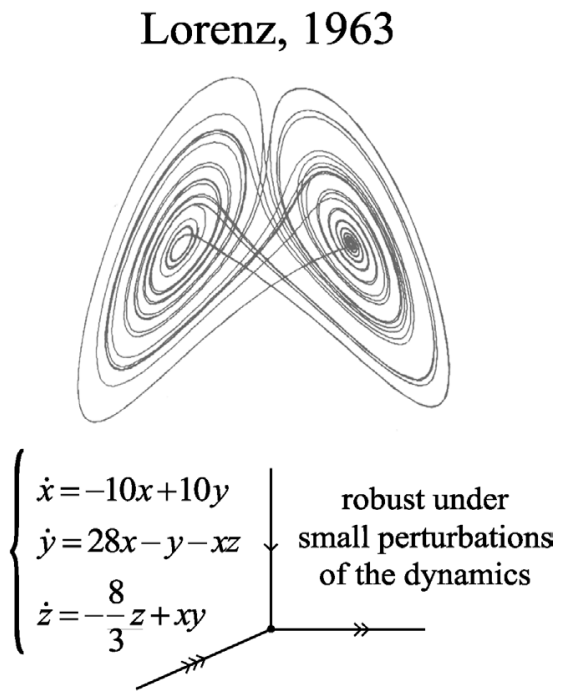

Fig. 2. 
periodic orbits. Actually, Lorenz focused on the property that the long range behaviour of the orbits in the basin of attraction is sensitive to initial conditions: with total probability, orbits with different initial conditions spread apart in the future as much as the diameter of the attractor. And he did so not with a rigorous mathematical proof, but with rather convincing computational arguments. Attractors with such characteristics are called Lorenz-like. Only, recently, Tucker [118] provided a computer aided proof that the original Lorenz's equations indeed corresponds to a sensitive (or chaotic) robustly transitive non-hyperbolic attractor containing the singularity $(0,0,0)$. A charming account of the Lorenz attractor's beautiful history is in Viana [123].

Only about ten years after Lorenz's work, a number of concrete Lorenz-like attractors were exhibited by Afraimovich, Bykov and Shil'nikov [2] and Guckenheimer and Williams [50], for which the authors provided mathematical proofs that they are sensitive to initial conditions and, moreover, robustly transitive and yet not hyperbolic. In the eighties Robinson [105] and Rychlik [108] showed that such attractors may be obtained, again in three dimensions, through bifurcations taking place in certain families of cubic differential equations - not quadratic, like above.

A new kind of attractor in three dimensions, the contracting Lorenz attractor or Lorenz-Rovella attractor, which is probability persistent but not robust, was obtained by Rovella [106] upon a previous work by Arneodo, Coullet and Tresser [11]: It contains a hyperbolic singularity with real eigenvalues (but now the sum of any two eigenvalues is negative), it is probability persistent in terms of Lebesgue probability or just probability persistent, but not robust (Fig. 3).

Another remarkable example is the one named after Hénon [52]. Like Lorenz, he provided ingenious computational arguments suggesting the existence of an attractor for a two-parameter family of quadratic diffeomorphisms of the plane. It would display a long range sensitivity of orbits near it with respect to their initial conditions. Hénon pointed to the existence of folds and expansion along lines in the attractor and a fractal structure in a transversal direction. The challenge was then to provide a formal proof of the existence of such a chaotic attractor with some degree of persistence.

\section{Lorenz-Rovella, 1992 \\ (Arneodo - Coullet - Tresser)}

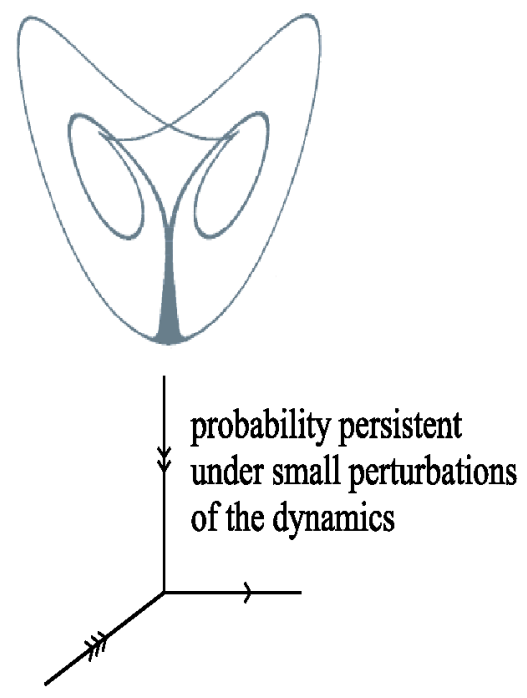

Fig. 3. 


\section{Hénon Attractor}

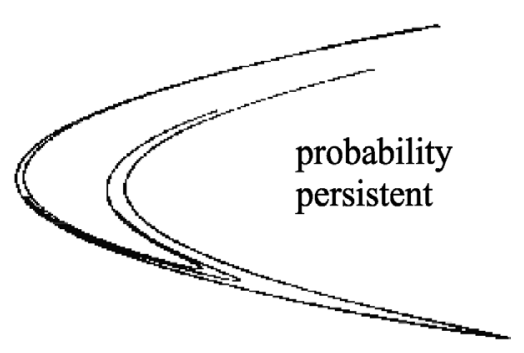

Fig. 4.

The equations and figure (Fig. 4) provided by Hénon were as follows:

$$
f_{a, b}(x, y)=\left(1-a x^{2}+y, b x\right), \quad \text { for } a \cong 1.4 \text { and } b \cong 0.3 .
$$

I also wish to mention Kolmogorov's pioneering and classic work on hydrodynamical attractors as presented by Arnold [14], as well as the charming paper of May on nonlinear dynamics and some applications [75].

By the last part of the seventies and going into the eighties, there appeared a string of very creative results giving rise to new insights on how dynamics could develop in the future. First, there was the work by Hénon mentioned above, proposing a new kind of attractor now named after him. Following that, there was the work by Feigenbaum [45] and in parallel Coullet and Tresser [37], concerning period doubling bifurcations for quadratic families of interval maps and the notable fact that the limiting ratio of approach of bifurcating parameter values converging to a limit point is a universal constant (independently of the specific quadratic family).

These works contributed to sparkle a much more robust development than before particularly of one real dimensional dynamics.

Then, there was the result of Jakobson [55], exhibiting new attractors of parametrized families of unimodal maps of the interval, including the quadratic family. They are probability persistent (but not robust) under small perturbations of the parameter. It was a key predecessor to the remarkable work by Benedicks and Carleson [23] showing the existence of a probability persistent Hénon-like attractor, which was obtained for the above coefficients (parameters) $a$ near 2 and $b$ small. Subsequently, Mora and Viana [79] showed that Hénon-like attractors occur in the unfolding of quadratic homoclinic tangencies associated to dissipative fixed or periodic hyperbolic points. As a consequence, the attractor appears near the values originally suggested by Hénon whose figure is displayed above, i.e. $a \approx 1.4$ and $b \approx 0.3$. This result was extended to higher dimensions, when the unstable manifold of the associated fixed or periodic point has dimension one [121], in the sectionally dissipative case as in Section 3.2. Also, Viana [122] exhibited new non-hyperbolic examples in higher dimensions which are, in fact, robust.

\subsection{Global conjecture on the finitude of attractors and their metric stability}

With the basic definitions, facts and examples presented above, we are now ready to formally state our main global conjecture, introduced in 1995 in a meeting in Paris in honor of A. Douady [90]. As mentioned in the abstract, we are considering the set of all $C^{r}(r \geqslant 1)$ dynamics, i.e. flows diffeomorphisms or transformations of a compact, smooth, boundaryless manifold or closed interval on the real line.

\section{Global Conjecture.}

- There is a dense set $D$ of dynamics such that any element of $D$ has finitely many attractors whose union of basins of attraction has total probability; 
- The attractors of the elements in $D$ support a physical (SRB) measure;

- For any element in $D$ and any of its attractors, for almost all small perturbations in generic $k$-parameter families of dynamics, $k \in \mathbb{N}$, there are finitely many attractors whose union of basins is nearly (Lebesgue) equal to the basin of the initial attractor; each such perturbed attractor supports a physical measure;

- Stochastic stability of attractors - the attractors of elements in $D$ are stochastically stable in their basins of attraction;

- For generic families of one-dimensional dynamics, with total probability in parameter space, the attractors are either periodic sinks or carry an absolutely continuous invariant measure.

One can also ask whether the first two items above are valid with total probability in parameter space.

\subsection{More on the existence of SRB probabilities - stochastic stability}

All the attractors that have been presented so far carry physical or SRB probability measures. Before we discuss some new results concerning other classes of dynamical systems whose attractors enjoy the same property, we introduce the notion of dominated decomposition.

\section{Dominated Decomposition - Definition}

Let $f$ be a $C^{r}$ map on $M$ and $\Lambda$ an invariant set for $f$. We say that $\Lambda$ has a dominated decomposition, see Fig. 5, if there exist $C>0$ and $0<\lambda<1$ such that $T_{\Lambda} M=E^{1} \oplus E^{2}, E^{1}$ and $E^{2}$ being $\mathrm{d} f$-invariant and

$$
\frac{\left\|\mathrm{d} f^{n}(x) v^{1}\right\|}{\left\|\mathrm{d} f^{n}(x) v^{2}\right\|} \leqslant C \lambda^{n} \frac{\left\|v^{1}\right\|}{\left\|v^{2}\right\|}, \quad \forall v=\left(v_{1}, v_{2}\right), v_{2} \neq 0, \forall n \geqslant 1 .
$$

In the figure, domination means that the angle between $\mathrm{d} f^{n}(x) v$ and $E^{2}$ decreases exponentially fast when $n \rightarrow \infty$. We say that $E^{2}$ dominates $E^{1}$ and that $f \mid \Lambda$ has $i$-dominated decomposition, where $1 \leqslant i<\operatorname{dim} M$, if $i=\operatorname{dim} E^{2}$.

In particular, we call $f$ partially hyperbolic when we have a decomposition $T_{\Lambda} M=E^{s} \oplus E^{c} \oplus E^{u}$, where $E^{s}$ is uniformly contracting, $E^{u}$ is uniformly expanding, $E^{u}$ dominates $E^{c s}=E^{s} \oplus E^{c}$ and $E^{c u}=E^{c} \oplus E^{u}$ dominates $E^{s}$. The definition can be adapted when $E^{u}=0, E^{s} \neq 0$ or $E^{s}=0, E^{u} \neq 0$.

Concerning SRB measures, recall that they were introduced by Sinai, Ruelle, and Bowen for hyperbolic systems, see Section 2.1. Such measures were shown to exist for probability persistent non-hyperbolic attractors: first, for interval maps by Jakobson in his pioneering work [55] and much more recently for Hénon-like attractors by Benedicks and Young [26]. In the latter case, subsequently, Benedicks and Viana [25] showed the remarkable fact that the SRB measure has full Lebesgue probability in a neighborhood of the attractor and it is unique.

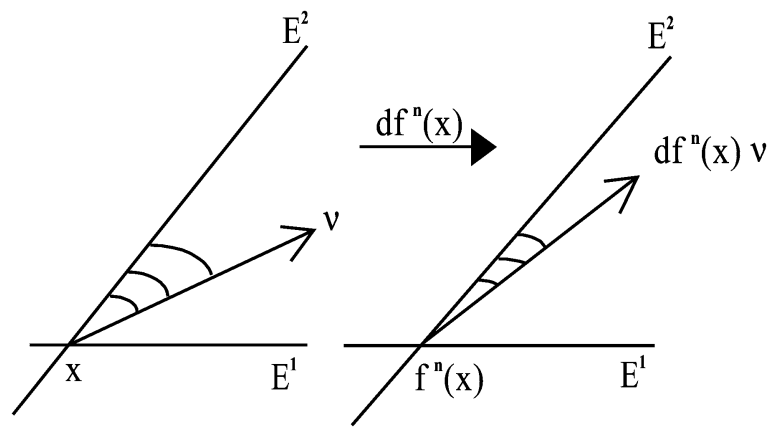

Fig. 5. 
On the other hand, SRB measures were constructed for Lorenz-like attractors by Bunimovich and Sinai [33] and Kifer [58], for Lorenz-Rovella attractors by Metzger [77], who also announced the same result for higher dimensional Lorenz attractors such as the ones constructed by Bonatti, Pumariño and Viana [31].

More recently, there were important developments on SRB measures for partially hyperbolic attractors, notably the following theorem due to Alves, Bonatti and Viana [5]:

Theorem. Let $K \subset M$ be a compact forward invariant set for a $C^{2}$ diffeomorphism on $M$. Suppose the subbundle $E^{c s}$ is uniformly contracting on $K$, and $E^{c u}$ is non-uniformly expanding on a positive Lebesgue measure set $H \subset K$. Then $H$ is covered mod 0, i.e., with total Lebesgue probability, by the basins of finitely many SRB measures.

The authors also introduced the notion of $\mathrm{cu}$-Gibbs states for an attractor $\Lambda$ with dominated decomposition $T_{\Lambda} M=E^{c s} \oplus E^{c u}$, after the pioneering work of Pesin and Sinai [100] on the existence of $u$-Gibbs states for an attractor $\Lambda$ with dominated decomposition $T_{\Lambda} M=E^{c s} \oplus E^{u}$. Notice that SRB measures are $u$-Gibbs states and, as a partial converse, ergodic $u$-Gibbs states with $k=\operatorname{dim} E^{c s}$ negative Lyapunov exponents are in fact SRB measures. Vasquez [120] extended considerably the theory of $c u$-Gibbs states and obtained results on their continuity with respect to the diffeomorphism, which he called statistical stability, as well as on the existence and finitude of SRB measures for diffeomorphisms with dominated decomposition. Before that, for certain parametrized families of diffeomorphisms, Dolgopyat [44] has even proved the differentiability of SRB measures, from which he inferred not only uniqueness but also good statistical properties for them.

It is also much worthwhile mentioning a recent important result by Tsujii [117]: partially hyperbolic surface endomorphisms of class $C^{r}$ with one-dimensional uniformly expanding subbundle and $r$ large enough, say $r \geqslant 19$, generically (residually) carry finitely many ergodic SRB measures whose union of basins of attraction has total Lebesgue probability. So, in this setting the first part of the main conjecture in the present paper is confirmed.

Actually, we believe that Tsujii's results can be generalized to higher dimensions for partially hyperbolic diffeomorphisms (maps) with one-dimensional central direction.

Problem. Show that generic partially hyperbolic maps as in Tsujii's work are stochastically stable in their basins of attraction.

Finally, there is the following relevant conjecture on SRB measures formulated by Viana in 1997:

Conjecture 1. Generically, non uniformly hyperbolic diffeomorphisms with a uniformly expanding subbundle carry finitely many SRB measures whose union of basins of attraction has total Lebesgue probability.

Concerning stability, let us emphasize, although not so surprisingly in view of Section 2.4, that none of the previous attractors are structurally stable, except for the hyperbolic ones. However, all of them are stochastically stable. For the hyperbolic case we refer to Kifer [57] and also Young [126]. For Hénon-like attractors the result is due to Benedicks and Viana [24]. For Lorenz and Lorenz-Rovella attractors it is due to Kifer [58] and Metzger [78], respectively.

\section{A strategy for proving the global conjecture on the finitude of attractors: to focus on homoclinic tangencies}

I believe that it might be possible to prove the main conjecture in this paper when there are no homoclinic tangencies in a robust way. At this point, a famous sentence by Poincaré in his classic treatise "Méthods Nouvelles de la Mécanique Céleste" comes to mind: "Rien n'est plus propre à nous donner une idée de la complication du 
problème des trois corps et en général de tous les problèmes de Dynamique ...”, referring to homoclinic orbits and the geometry of the corresponding stable and unstable manifolds. More concretely, we shall mention in the sequel that a robust absence of homoclinic tangencies implies, at least in the $C^{1}$ topology, a certain amount of hyperbolicity, namely a dominated decomposition. Since hyperbolic systems satisfy the main conjecture, this may also be the case here. And, if so, we could concentrate our efforts in the fundamental and very difficult problem of understanding the dynamics arising from unfolding homoclinic or heteroclinic cycles, as much as the attractors and their basins are concerned.

This will be discussed in the sequel. Let us first see what is known in terms of a weak form of hyperbolicity, in the present case dominated decomposition, when robustly we have no homoclinic tangencies.

Theorem. Let $f$ be a diffeomorphism and let $\Lambda$ be a compact, maximal invariant set with a dense subset of hyperbolic periodic orbits of unstable index $i>0$. Suppose that there are no homoclinic tangencies associated to periodic hyperbolic orbits with unstable index $i$ in any $C^{1}$ small continuation of $\Lambda$. Then, there is an $i$-dominated decomposition of $f \mid \Lambda$.

Let us recall the meaning of continuation of a maximal invariant set in the statement of the theorem. Let $\Lambda=$ $\bigcap_{n} \in \mathbb{Z} f^{n}(U)$, where $U$ is a neighborhood of $\Lambda$ and let $\mathcal{U}$ be a $C^{1}$ neighborhood of $f$. For $g \in \mathcal{U}$, we call $\Lambda_{g}=\bigcap_{n \in \mathbb{Z}} g^{n}(U)$ the $g$-continuation of $\Lambda$.

This theorem is due to Pujals and Sambarino [102] in dimension two and to Wen [124] in higher dimensions. It is certainly relevant to pursue a proof of the main conjecture in such a setting:

Conjecture 2. There is a residual (or at least dense) subset $R$ of $C^{r}$ diffeomorphisms, $r \geqslant 1$, such that: If $f \in R$ and $\Lambda$ is a maximal invariant set as in the theorem above, with no homoclinic tangencies associated to periodic points in a robust way, then $f$ exhibits only finitely many attractors inside $\Lambda$. Such attractors are stochastically stable.

A particularly interesting case in this direction is when $f, \Lambda$ are as before, but now it is assumed that the periodic points with biggest unstable index in $\Lambda$ are dense in it. These conjectures can in particular also be posed for partially hyperbolic pairs $f, \Lambda$; see Conjecture 1 in Section 2.8 .

\subsection{Bifurcation theory}

We now turn to bifurcation theory, a classical and central area in dynamics, as set forth by Poincaré. The list of contributions is very notable and we shall highlight some of them:

- Birkhoff [27] in the 30's has shown that transversal homoclinic orbits for surface diffeomorphisms are accumulated by periodic orbits (so, infinitely many of them). About three decades later, Smale [113,114] went much further by showing that they are part of what he called a horseshoe in any dimension. Before, in the 20's, there was the remarkable work by Van der Pol on relaxation oscillations [40] to model radio transmissions and electric circuits, among other applications. Following up this pioneer work, there were a series of papers by Cartwright and Littlewood [34,35], Littlewood [68,69] and Levinson [65], in the 40's and 50's in which Van der Pol's equations with parameters were considered and new kind of solutions were found, going through dynamic bifurcations when varying parameters. In his autobiography, Littlewood called his work in this topic "the monster", since he seemed to continuously work hard but without a clue of at which port he would arrive if any ... Then, in the late 70's and early 80's, Levi revisited the problem disposing of new developments that were not at the time available to Littlewood, such as the horseshoe, sometimes with small and sometimes large Hausdorff dimensions, Newhouse's infinitely many simultaneous sinks and other intricate phenomena that are created when unfolding (bifurcating) quadratic homoclinic tangencies; see Section 2.5. He showed [64] that all 


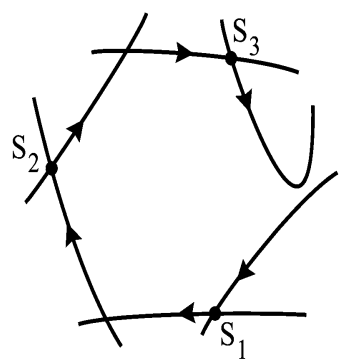

No cycle

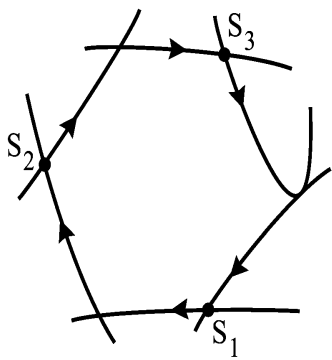

Creating an unstable cycle

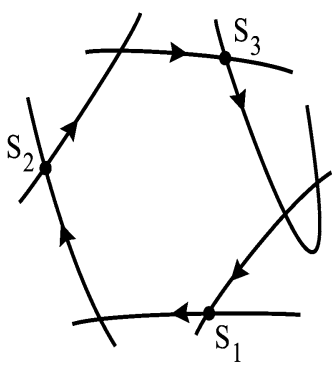

A stable cycle

Fig. 6.

such phenomena were present in cross-sections to 3-dimensional flows generated by Van der Pol's equations. Such equations which were motivated by applications, clearly led to the development of deep mathematics.

- Closely following Poincaré's fundamental work, Andronov and school [6-8] set the ground for bifurcation theory in dynamics, influencing generations to come, noteworthily his work with Pontrjagin on structural stability that has been already mentioned.

- In the 60's and 70's, Arnold and school were a source of fine questions and results, in particular much extending the previous work on bifurcations, as can be seen in [12] and [13]. I wish to add here the work of Il'Yashenko and co-authors, as in [53].

- Much relevant has also been the work of Shil'nikov and school, mostly devoted to bifurcation theory and more particularly to the unfolding of homoclinic tangencies and creation of horseshoes [3,4] and [110].

- An extensive study of bifurcations and (structural) stability of parametrized families of dynamics were performed in the seventies and going through the next two decades by Newhouse, Palis, Takens $[87,88,92,93]$ and Dias Carneiro [38] and Viana, Yoccoz, Moreira [83,84,96,97], whom are also referred to in the sequel on topics discussing homoclinic bifurcations.

See also $[15,28,53,95]$ for more results and further references.

We have generally been baffled by the creation of cycles in dynamics, in particular 1-cycles, which correspond to homoclinic tangencies; a 3-cycle is in Fig. 6. Actually, the creation of cycles is often unavoidable when we consider parametrized families of systems and vary the parameters. In most cases, the unfolding of cycles gives rise to a very complicated, chaotic evolution of the dynamics in terms of parameters. In particular, they are a source of counter-examples to the denseness of hyperbolicity in the space of all dynamical systems endowed with any $C^{r}$ topology, $r \geqslant 1$ (the only unknown case being that of $C^{1}$ diffeomorphisms on surfaces, see Ures [119]).

Still, we expect for a dense set of parameters to have only finitely many attractors for the corresponding dynamical systems. In fact, the same may be true with total Lebesgue probability in the space of parameters.

\subsection{Unfolding homoclinic tangencies}

In the sentence at the beginning of Section 3, Poincaré was referring to transversal homoclinic orbits of surface diffeomorphisms. One can imagine that he would possibly be as enthusiastic with respect to Birkhoff and Smale's results, mentioned in Section 3.1. They highlight even more so the richness of the dynamics implied, in all dimensions, by the presence of a transversal homoclinic orbit. Indeed, Smale's horseshoe corresponded to a new prototype dynamical model: its maximal invariant set is like the product of Cantor sets in which the periodic orbits are dense. 

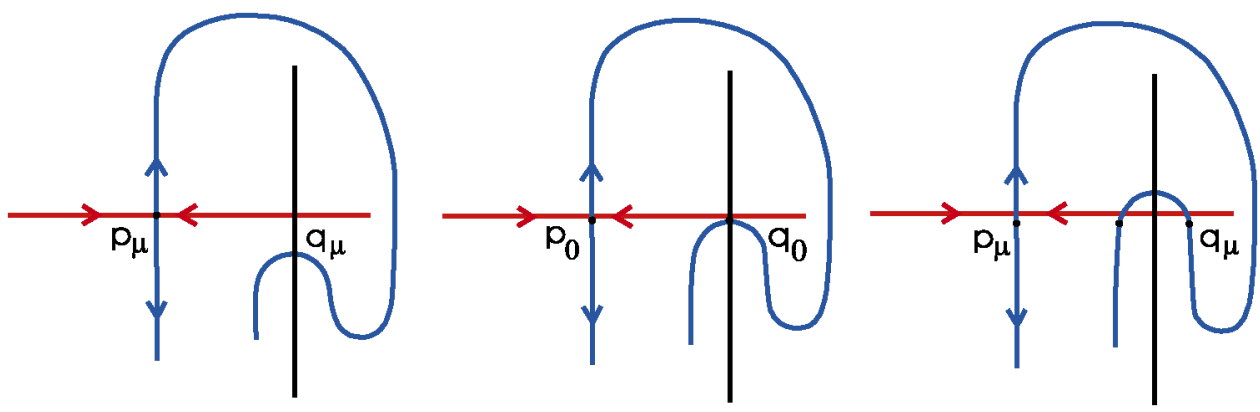

Fig. 7.

Similarly striking is the following list of complex phenomena discovered in the last three and half decades. It concerns the unfolding of a homoclinic tangency $q_{0}$ associated to a fixed (periodic) orbit $p_{0}$ with positive speed at $\mu=0$, for a $C^{2}$ family $f_{\mu}, \mu$ small, of surface diffeomorphisms:

- There are infinitely many simultaneous sinks residually in some open subsets of parameter values, as shown by Newhouse $[85,86]$. This renowned result was extended to higher dimensions by Palis and Viana [96], when $W^{u}\left(p_{0}\right)=1$ and $p_{0}$ is sectionally dissipative, i.e., the product of any two different eigenvalues of $\mathrm{d} f_{0}$ has norm smaller than one.

- There are Hénon-like attractors for some parameter values, due to Mora and Viana [79], extending Benedicks and Carleson [23], and to Viana [121] in general in the codimension-one and sectionally dissipative case.

- There are infinitely many simultaneous Hénon-like attractors for dense subsets of open sets of parameter values, as shown by Colli [36].

- A fast growth of the number of periodic points occurs residually in an open Newhouse set as above, due to Kaloshin and Hunt [56], based on Shil'nikov, Gonchenko and Turaev [47].

In view of these dynamical phenomena, one is lead to consider the question of whether the coexistence of infinitely many sinks or Hénon-like attractors or just attractors are probability persistent, i.e., they can occur with positive Lebesgue probability in parameter space:

Conjecture 3. In the unfolding of a homoclinic tangency, the coexistence of infinitely many sinks or, more generally, attractors is not a probability persistent phenomenon. In other words, with total probability in the parameter line, the corresponding maps exhibit only finitely many attractors, in particular sinks, in a neighborhood of the orbit of $q_{0}$ and $p_{0}$ (see Fig. 7).

A very nice recent result concerning this conjecture for surface diffeomorphisms is due to Gorodetski and Kaloshin [48], much extending Tedeschini and Lalli and Yorke [116]. It says that, for any positive integer N, infinitely many coexisting localized sinks with cyclicity bounded by $N$ or with period relatively large with respect to $N$ is not probability persistent. To understand this result, we need to introduce the definition of a localized sink and its cyclicity.

Consider Fig. 8, where $q_{0}$ is a homoclinic tangency, say quadratic, associated to the fixed (periodic) hyperbolic point $p_{0}$ and let $V$ and $U$ be fixed small disjoint neighborhoods of the segments $\left[f_{0}\left(q_{0}\right), p_{0}\right],\left[p_{0}, f_{0}^{-1}\left(q_{0}\right)\right]$ in the stable and unstable manifolds and of $p_{0}$, respectively.

Let $\Lambda_{\mu}=\bigcap_{n \in \mathbb{Z}} f_{\mu}^{n}(V \cup U)$.

We can now define a periodic sink for $f_{\mu}$ as being localized if it belongs to $\Lambda_{\mu}$. In such a case, it's called $\left(\Lambda_{\mu}, s\right)$-localized if it visits $U$ exactly $s$ times during its minimal period. Then, we call $s$ its cyclicity. 


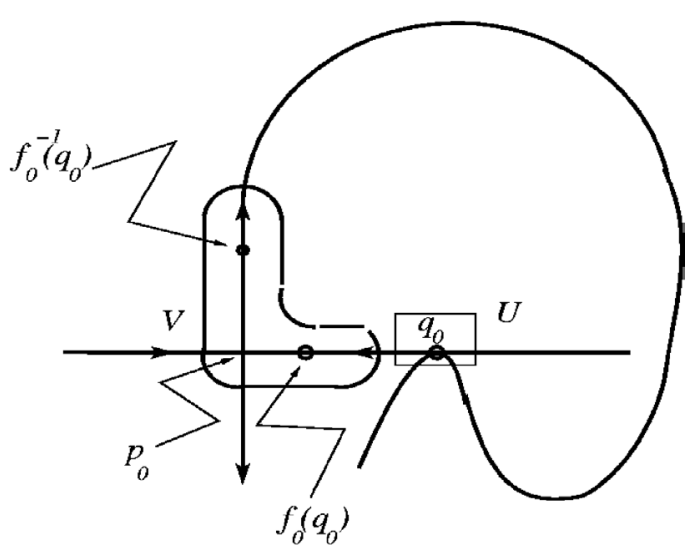

Fig. 8.

We close for the moment the discussion on the unfolding of homoclinic tangencies. We shall return to it in Section 3.4, but from a somewhat different point of view. We shall consider homoclinic tangencies associated to fixed or periodic orbits, that may or not be part of a larger hyperbolic set, say $K$, at the initial parameter value for the unfolding. We shall see that the Hausdorff dimension of $K$ is a crucial ingredient in establishing some prevalence of hyperbolicity or non-uniform hyperbolicity for the dynamics, in terms of the parameter near its initial value.

\subsection{More on the dichotomy hyperbolicity - homoclinic bifurcations}

Along the lines discussed at the introduction to the present section, we have set up the following conjecture, which we consider by itself relevant:

Conjecture 4. In any dimension, every diffeomorphism can be $C^{r}$ approximated, $r \geqslant 1$, by a hyperbolic one or one exhibiting either a homoclinic tangency or a (finite) cycle of hyperbolic periodic orbits with different stable dimensions - called a heterodimensional cycle $[28,87]$.

As mentioned, hyperbolic diffeomorphisms are not dense (except for the circle and unknown for $r=1$ on twodimensional surfaces). What the conjecture states is that it is enough to complement them with diffeomorphisms exhibiting just two different dynamical structures, which are relatively simple to formulate. Yet, such diffeomor-

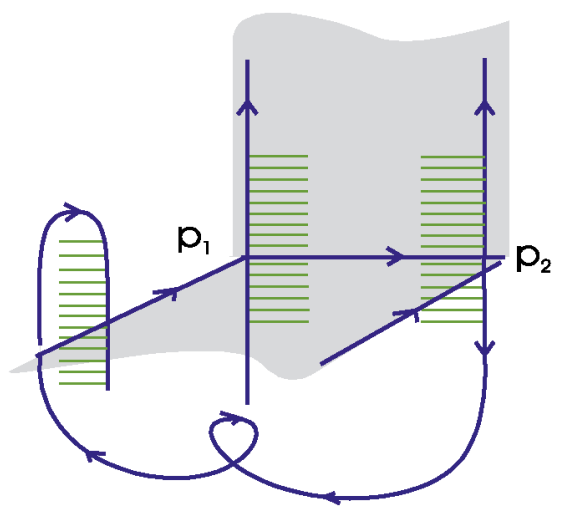

Fig. 9. 
phisms display an array of varying intricate dynamics when subject to bifurcations, say in parametrized form. They may be in fact at a door of the difficult realm of dynamics needed to be better understood. Let me point out that there is a number of impressive results concerning heterodimensional cycles due to Bonatti, Diaz, Pujals and Rocha, like the ones in [30,41,43], also presented in [28].

We have a version of Conjecture 4 for flows. To state it, we have to consider the notion of a singular cycle, introduced in [63] and [22]. Here, a singular cycle for a flow just means a cycle involving singularities, at least one of them, and periodic orbits. Similar to the diffeomorphism case, a heterodimensional cycle for a flow is a cycle involving finitely many hyperbolic periodic orbits with different stable dimensions (indices).

Conjecture 5. In any dimension, every flow (vector field) can be $C^{r}$ approximated by a hyperbolic one or by one displaying a homoclinic tangency or a singular cycle or a heterodimensional cycle.

The latter can only occur in dimensions greater than three. In dimension three, we can demand even more:

Conjecture 6. Every three-dimensional flow can be $C^{r}$ approximated by a hyperbolic one or by one displaying a singular cycle or a Lorenz-like attractor or repellor.

In this spirit, Conjecture 5 may be formulated in a somewhat stronger form, substituting singular cycle by singular hyperbolic set in the sense of [80,81] and [82].

These conjectures have been the subject of much interest and some remarkable results have been obtained in the $C^{1}$ context. First of all, a breakthrough was due to Pujals and Sambarino [102] when proving Conjecture 4 for $C^{1}$ surface diffeomorphisms. Just now, Pujals is presenting in a long pre-publication [101] a similar fact for three-dimensional diffeomorphisms restricted to contracting regions. Partial advancements were provided by Wen $[124,125]$ and an announcement has been made by Hayashi.

Concerning the conjectures for flows, Arroyo and Rodriguez and Hertz [16] provided a proof of Conjecture 5 in dimension three. In particular, they made an ingenious use of the work of Pujals-Sambarino mentioned above. Conjecture 6 relative to flows in dimension three remains open.

We conclude this part by very briefly mentioning some outstanding results relative to an important topic, certainly most relevant to our discussion at the beginning of this section. We shall do that first in the context of diffeomorphisms and then flows.

Let $\Lambda$ be a maximal (in some neighborhood of it) invariant, transitive set for a diffeomorphism $f$ on a closed (as usual here) manifold $M$. We say that $\Lambda$ is robustly transitive if $\Lambda_{g}$ is transitive, where $\Lambda_{g}$ is the continuation of $\Lambda$ and $g$ is any map $C^{1}$ close to $f$. Similarly for flows.

Assume $\Lambda$ is robustly transitive. Then:

- For $C^{1}$ diffeomorphisms, first, Mañé [72] has shown that if $\operatorname{dim} M=2$, then $\Lambda$ is a hyperbolic set. Then, in the three-dimensional case, $\Lambda$ has to be partially hyperbolic, a result due to Diaz, Pujals and Ures [42], and in higher dimensions, $\Lambda$ has at least to display a dominated decomposition, as proved by Bonatti, Diaz and Pujals [29].

- For $C^{1}$ flows, Morales, Pacifico and Pujals [82] proved the striking fact that on 3-manifolds a robustly transitive set is a Lorenz-like attractor or reppeler.

\subsection{Homoclinic bifurcations and Hausdorff dimension}

We have now a somewhat better understanding of the (complicated) dynamics that arises when we unfold homoclinic tangencies, say associated to a hyperbolic set $\Lambda$. Still, we have to go much further to be able, for instance, to respond to the challenge represented by Conjecture 3 in Section 3.2. Already a major question is 


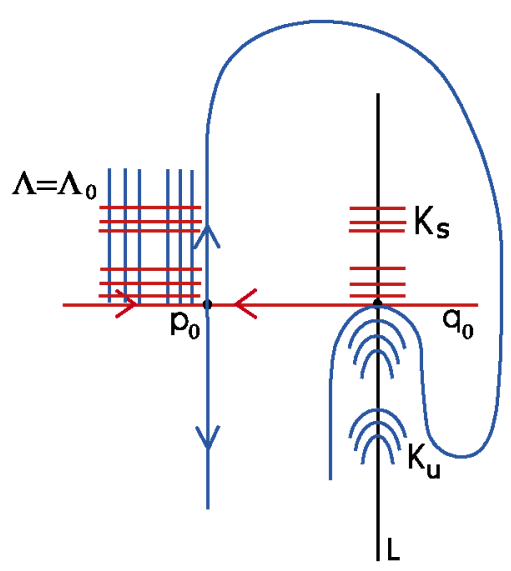

Fig. 10 .

whether densely in the parameter line the corresponding maps have only finitely many localized attractors, i.e., in a neighborhood of $p_{0}$ and of the orbit of $q_{0}$, as in figure in Section 3.2.

So, let us again consider the unfolding with positive speed at $\mu=0$ of a quadratic homoclinic tangency $q_{0}$ for a $C^{2}$ family $f_{\mu}$ of diffeomorphisms, associated to a fixed (periodic) point $p_{0}$ of a hyperbolic set $\Lambda=\Lambda_{0}$. We take $\mu$ small and let $\Lambda_{\mu}$ be the continuation of $\Lambda$. Also, let $K_{s}, K_{u}$ be Cantor sets obtained by intersecting (for the first time from points in $\Lambda$ ) the leaves of the stable and unstable foliations of $\Lambda$ with $L$, where $L$ is a line transversal to the stable manifold of $p_{0}$. One may consider $L$ as the unfolding parameter line. See Fig. 10. Finally, let $d_{s}$ and $d_{u}$ be the Hausdorff dimensions of $K_{s}$ and $K_{u}$, respectively. Notice that $H D(\Lambda)=d_{s}+d_{u}$, where $H D(\Lambda)$ is the Hausdorff dimension of $\Lambda$.

We have:

- If $H D(\Lambda)<1$, then hyperbolicity is fully prevalent at $\mu=0$. That is, the set of parameter values corresponding to which $f_{\mu}$ is hyperbolic has Lebesgue density one at $\mu=0$, Newhouse and Palis [87] and Palis and Takens $[93,94]$.

- If $H D(\Lambda)>1$, then hyperbolicity is not fully prevalent at $\mu=0$, Palis and Yoccoz [97].

- If $H D(\Lambda)>1$, then the arithmetic difference $K_{s}-K_{u}$ robustly contains non-trivial intervals. From that, one infers that homoclinic tangencies for $f_{\mu}$ have positive density at $\mu=0$, implying the previous result in a strong form, Moreira and Yoccoz [84].

Notice that when $H D(\Lambda)<1$, then $K_{s}-K_{u}$ has Lebesgue measure equals to zero.

From this string of facts, one can state the following dichotomy principle concerning the unfolding on surfaces of a homoclinic tangency associated to a hyperbolic set $\Lambda$ :

The hyperbolic maps in the parametric family are prevalent if and only if $H D(\Lambda)<1$.

Such a dichotomy was suggested by the author about two decades ago.

The most recent result in this direction is the following one, going quite deeply into the dynamical structure of $f_{\mu}$ after the unfolding of the homoclinic tangency:

- If $\left(d_{s}+d_{u}\right)^{2}+\left(\max \left(d_{s}, d_{u}\right)\right)^{2}<d_{s}+d_{u}+\max \left(d_{s}, d_{u}\right)$, then non-uniform hyperbolicity is fully prevalent at $\mu=0$. In particular, the set of parameter values corresponding to which the maps $f_{\mu}$ have localized attractors 
is of density zero at $\mu=0$, Palis and Yoccoz [98]. Localized here has the same meaning as in Gorodetski and Kaloshin's result in Section 3.2, but now $V$ is a neighborhood the whole hyperbolic set $\Lambda$ and $U$ is as before.

In our present proof of the last result, outlined in [98] and the full proof soon to appear, we treat the unfolding of a quadratic tangency between stable and unstable manifolds of two different periodic orbits in the hyperbolic set $\Lambda$. But, certainly the same should be true in the homoclinic case. We observe, from such a proof, that for many parameter values (total density at $\mu=0$ ), the continuation of the forward maximal invariant set in a neighborhood $U \cup V$ as above has Hausdorff dimension smaller than two. Similarly, for the continuation of the backward maximal invariant set. Notice that, for such parameter values, there may be tangencies between stable and unstable manifolds for the corresponding maps, but no attractors (nor repellors).

Let us add a few words about the proof. It goes by showing that, for most parameters, the limit set is hyperbolic, in a delicate, non-uniform sense. Essentially, although the limit set may contain tangencies, they correspond to very special points: at "most" points there are transversal directions which are (asymptotically) contracted by forward and backward iterates, respectively. The proof of this fact requires a very careful analysis of how trajectories return close to the tangencies and, even, the very definition of what a "tangency" is. To ensure hyperbolic behavior, such returns should not be too frequent nor too close. This is achieved by parameter exclusions, which turns out to be less and less significant near the original tangency parameter (Lebesgue density zero). The rate of formation of tangencies is a crucial ingredient, and it is closely related to the Hausdorff dimension of the original horseshoe. The assumption, that this dimension is not far from one, ensures that the number of tangencies that must be considered at each stage grows fairly slowly. This implies that a fairly small amount (in measure) of parameter exclusions is needed. Returns close to the tangencies yield quadratic type folds. The condition on the frequency and depth of returns is used to ensure that folds always are "ironed-out" before a new return occurs. In this way, one never has to deal with contacts of order bigger than two.

One can certainly expect to have a similar general result without imposing any restriction on the Hausdorff dimension of the original horseshoe, at the price of having to deal with higher order contacts.

On the horizon lies the case of area preserving maps. An example is the famous family of standard maps on the torus, for which the limit set is the whole ambient space and, thus, has Hausdorff dimension two:

$$
f_{\mu}(x, y)=(-y+2 x+\mu \sin (2 \pi x), x) .
$$

In this case, one has to study contacts of all order simultaneously. The main open question concerning this family is whether there exists a positive Lebesgue measure set of parameter values $\mu$, for which the corresponding maps are ergodic and non-uniformly hyperbolic.

\subsection{Unfolding homoclinic tangencies in higher dimensions}

To have a grasp of typical dynamics in all dimensions, we certainly must pursue a deep study of the unfolding of homoclinic tangencies in such a generality.

In doing so, we shall face some new and serious difficulties in comparison with the two-dimensional case. One of them is that in general the stable and unstable foliations of hyperbolic sets, when having codimension bigger than one, are not differentiable or even Lipshitz, independenty of the smoothness of the dynamics. As a consequence, the invariance along leaves of such foliations of some key instruments of nowadays dynamics, like Hausdorff dimension, are simply no longer available in higher dimensions. This is because at least one of the foliations must have codimension bigger than one.

Still, it's a promising fact that the obstacles, such as the one just mentioned, have been in some cases successfully surpassed. It was so in several of the results in this paper, particularly in the extension of Newhouse's infinitely many sinks phenomenon to higher dimensions in Section 3.2. Here, we comment on a recent result that goes much in the direction we are conveying. 
As before, we consider one-parameter families of diffeomorphisms $f_{\mu}$ of class $C^{2}, \mu \in(-1,1)$, that generically (positive speed in $\mu$ at $\mu=0$ ) unfold a quadratic homoclinic tangency $q_{0}$ associated to a hyperbolic horseshoe $\Lambda=\Lambda_{0}$.

A reasonable hypothesis, as done on surfaces, see Section 3.4, is to assume that $f_{\mu}$ is hyperbolic (Axiom A) for $\mu<0$. So, we are creating a cycle, a homoclinic tangency, at $\mu=0$. This hypothesis implies that the weak stable and unstable eigenvalues of $\mathrm{d} f_{0}$ at $p_{0}$ are well defined and that they are real numbers. Moreira, Viana and myself then showed (see a résumé in [83]) that the dichotomy

hyperbolicity is prevalent $\Leftrightarrow$ Hausdorff dimension $<1$

is true in all dimensions.

Prevalence of hyperbolicity means: the set of $\mu$ 's for which hyperbolicity holds for the maximal invariant set of $f_{\mu}$ in a neighborhood of $\Lambda$ and $q_{0}$ as in Section 3.4, has total density at $\mu=0$. And by Hausdorff dimension smaller than one, we mean $H D_{s}(\Lambda)+H D_{u}(\Lambda)<1$ where

$$
H D_{s}(\Lambda)=H D\left(\Lambda \cap W^{s}\left(p_{0}\right)\right), \quad H D_{u}(\Lambda)=H D\left(\Lambda \cap W^{u}\left(p_{0}\right)\right) .
$$

Formally, we state the result as follows:

There are open sets $R_{1}$ and $R_{2}$ of one-parameter families of $C^{k}$ diffeomorphisms, $k \geqslant 2$, unfolding a homoclinic tangency as set above, such that

(a) $R_{1} \cup R_{2}$ is dense;

(b) for families in $R_{1}$, we have

$$
H D_{S}(\Lambda)+H D_{u}(\Lambda)<1 \text { and } \lim _{\delta \rightarrow 0} \frac{m(H \cap[0, \delta])}{\delta}=1
$$

where $H=\left\{\mu \mid f_{\mu}\right.$ is hyperbolic $\}$ and $m$ stands for Lebesgue measure;

(c) for families in $R_{2}$, we have for $\delta>0$

$$
H D_{s}(\Lambda)+H D_{u}(\Lambda)>1 \text { and } \liminf _{\delta \rightarrow 0} \frac{m\left(T_{s} \cap[0, \delta]\right)}{\delta}>0
$$

where $T_{s}=\left\{\mu \mid f_{\mu}\right.$ presents a persistent homoclinic tangency associated to $\left.\Lambda_{\mu}\right\}$ and $\Lambda_{\mu}$ is the continuation of $\Lambda, \mu$ small.

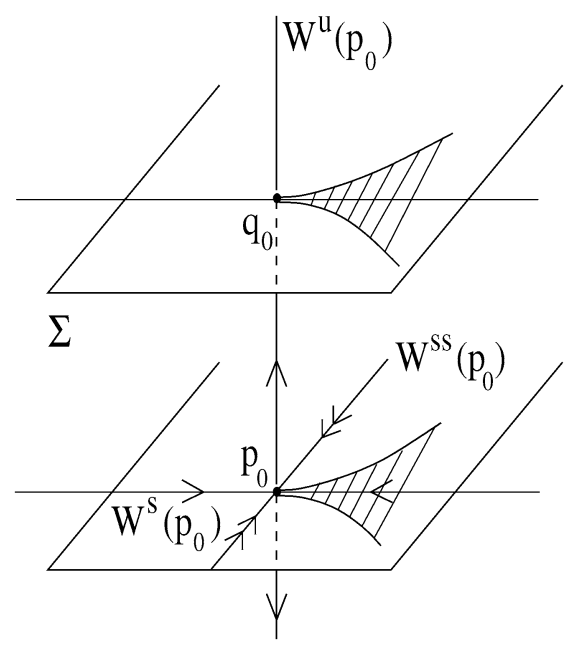

Fig. 11. 
We briefly comment the proof of this result:

Part (b) is reminiscent of the Palis-Takens' result on surfaces [94]. The proof here goes by ensuring that, up to small perturbation, the horseshoe avoids the strong stable and unstable directions. This is possible because its Hausdorff dimension is small. It implies that $\Lambda \cap W^{s}(p)$ is contained in a cuspidal region around the weak stable direction of $p_{0}$. Similarly for $\Lambda \cap W^{u}(p)$ with respect to the weak unstable direction of $p_{0}$. See Fig. 11. It has the geometric consequence that the horseshoe is roughly two-dimensional. This permits to mimic the two-dimensional arguments to obtain uniform transversality of stable and unstable foliations of the continuation of the original horseshoe for most parameters.

Part (c) is considerably more delicate. The main ingredient is the construction, after perturbation of $f$, of strong-stable and strong-unstable foliations of codimension-one for hyperbolic subsets of $\Lambda$ with almost the same Hausdorff dimensions.

These foliations are used to reduce the study of the geometries of the stable and unstable foliations near the initial homoclinic tangency to the two-dimensional case. Another key step is to obtain stable tangencies, in terms of parameters, of stable and unstable manifolds of periodic orbits as in Moreira and Yoccoz [84].

\section{References}

[1] R. Abraham, S. Smale, Nongenericity of $\Omega$-stability, in: Global Analysis, Berkeley 1968, in: Proc. Sympos. Pure Math., vol. XIV, Amer. Math. Soc., 1970.

[2] V.S. Afraimovich, V.V. Bykov, L.P. Shil'nikov, On the appearance and structure of the Lorenz attractor, Dokl. Acad. Sci. USSR 234 (1977) 336-339.

[3] V.S. Afraimovich, L.P. Shil'nikov, The accessible transitions from Morse-Smale systems to systems with several periodic motions, Izv. Akad. Nauk SSSR 38 (1974) 1248-1288; English translation: V.S. Afraimovich, L.P. Shil'nikov, Math. USSR-Izv. 8 (1976) 1325-1370.

[4] V.S. Afraimovich, L.P. Shil'nikov, On bifurcations of codimension 1, leading to the appearance of fixed points of a countable set of tori, Dokl. Akad. Nauk SSSR 262 (1982) 777-780; English translation: V.S. Afraimovich, L.P. Shil'nikov, Soviet Math. Dokl. 25 (1982) 101-105.

[5] J.F. Alves, C. Bonatti, M. Viana, SRB measures for partially hyperbolic systems whose central direction is mostly expanding, Invent. Math. 140 (2000) 351-398.

[6] A. Andronov, E.A. Leontovich, Some cases of dependence of limit cycles on parameters, Uchen. Zap. Gor'kov. Univ. 6 (1939) 3-24.

[7] A. Andronov, E.A. Leontovich, I.I. Gordon, A.G. Majer, Qualitative Theory of Dynamical Systems of Second Order, Nauka, Moscow, 1966, p. 569.

[8] A. Andronov, E.A. Leontovich, I.I. Gordon, A.G. Majer, The Theory of Bifurcations of Dynamical Systems on the Plane, Nauka, Moscow, 1967, p. 487.

[9] A. Andronov, L. Pontryagin, Systèmes grossiers, Dokl. Akad. Nauk USSR 14 (1937) 247-251.

[10] D.V. Anosov, Geodesic flows on closed Riemannian manifolds of negative curvature, Proc. Steklov Math. Inst. 90 (1967) 1-235.

[11] A. Arnéodo, P. Coullet, C. Tresser, Possible new strange attractors with spiral structure, Comm. Math. Phys. 79 (1981) $673-679$.

[12] V.I. Arnold, Small denominators I: On the mapping of a circle to itself, Izv. Akad. Nauk Math. Ser. 25 (1961) 21-86; English translation: V.I. Arnold, Transl. Amer. Math. Soc. 46 (1965) 213-284.

[13] V.I. Arnold, Lectures on bifurcations and versal families, Uspekhi Math. Nauk 27 (1972) 119-184; English translation: V.I. Arnold, Russian Math. Surveys 27 (1973) 54-123.

[14] V.I. Arnold, Kolmogorov's hydrodynamics attractors. turbulence and stochastic processes: Kolmogorov's ideas 50 years on, Proc. Roy. Soc. London Ser. A 434 (1991) 19-22;

English translation: V.I. Arnold, Russian Math. Surveys 27 (1973) 54-123.

[15] V.I. Arnold, V.S. Afraimovich, Yu. Ilyasheko, L.P. Shilnikov, Bifurcation Theory and Catastrophe Theory, Springer, 1999.

[16] A. Arroyo, F. Rodriguez Hertz, Homoclinic bifurcations and uniform hyperbolicity for three-dimensional flows, Ann. Inst. H. Poincaré 20 (2003) 805-841.

[17] A. Avila, M. Lyubich, W. de Melo, Regular or stochastic dynamics in real analytic families of unimodal maps, Invent. Math. 154 (2003) 451-550.

[18] A. Avila, C.G. Moreira, Phase-parameter relation sharp statistical properties of unimodal maps, Contemp. Math., in press.

[19] A. Avila, C.G. Moreira, Statistical properties of unimodal maps: physical measures, periodic orbits and pathological laminations, Publ. Math. IHES, in press.

[20] A. Avila, C.G. Moreira, Statistical properties of unimodal maps: the quadratic family, Ann. of Math., in press. 
[21] A. Avila, C.G. Moreira, Statistical properties of unimodal maps: smooth families with negative Schwarzian derivative, Astérisque 286 (2003) 81-118.

[22] R. Bamón, R. Labarca, R. Mañé, M.J. Pacifico, The explosion of singular cycles, Publ. Math. IHES 78 (1993) $207-232$.

[23] M. Benedicks, L. Carleson, The dynamics of the Hénon map, Ann. of Math. 133 (1991) 73-169.

[24] M. Benedicks, M. Viana, Random perturbations and statistical properties of Hénon-like maps, Ann. Inst. H. Poincaré Anal. Non Linéaire.

[25] M. Benedicks, M. Viana, Solution of the basin problem for Hénon-like attractors, Invent. Math. 143 (2001) 375-434.

[26] M. Benedicks, L.-S. Young, SBR-measures for certain Hénon maps, Invent. Math. 112 (1993) 541-576.

[27] G.D. Birkhoff, Nouvelles recherches sur les systèmes dynamiques, Mem. Pont. Acad. Sci. Novi. Lyncaei 1 (1935) 85-216.

[28] C. Bonatti, L. Diaz, M. Viana, Dynamics Beyond Uniform Hyperbolicity, Encyclopaedia Math. Sci., vol. 102, Springer, 2004.

[29] C. Bonatti, L.J. Díaz, E. Pujals, A $C^{1}$-generic dichotomy for diffeomorphisms: weak forms of hyperbolicity or infinitely many sinks or sources, Ann. of Math. 158 (2003) 355-418.

[30] C. Bonatti, L.J. Díaz, E. Pujals, J. Rocha, Robust transitivity and heterodimensional cycles, Astérisque 286 (2003) $187-222$.

[31] C. Bonatti, A. Pumariño, M. Viana, Lorenz attractors with arbitrary expanding dimension, C. R. Acad. Sci. Paris, Sér. I Math. 325 (1997) $883-888$

[32] R. Bowen, D. Ruelle, The ergodic theory of Axiom A flows, Invent. Math. 29 (1975) 181-202.

[33] L.A. Bunimovich, Ya.G. Sinai, Stochasticity of the attractor in the Lorenz model, in: Nonlinear Waves, in: Proc. Winter School, Nauka, Moscow, 1980, pp. 212-226.

[34] M. Cartwright, J. Littlewood, On non-linear differential equations of the second order, J. London Math. Soc. 20 (1945) $127-153$.

[35] M. Cartwright, J. Littlewood, On non-linear differential equations of the second order, Ann. of Math. 48 (1947) $472-494$.

[36] E. Colli, Infinitely many coexisting strange attractors, Ann. Inst. H. Poincaré Anal. Non Linéaire 15 (1998) 539-579.

[37] P. Coullet, C. Tresser, Itérations d'endomorphims et groupe de renormalization, C. R. Acad. Sci. Paris, Sér. I 287 (1978) $577-580$.

[38] M.J. Dias Carneiro, J. Palis, Bifurcations and global stability of families of gradients, Publ. Math. IHES 70 (1989) $70-163$.

[39] W. de Melo, Structural stability of diffeomorphisms on two-manifolds, Invent. Math. 21 (1973) 233-246.

[40] B. Van der Pol, On relaxation oscillations, Philos. Mag. Ser. 72 (1926) 978-992.

[41] L.J. Díaz, Robust nonhyperbolic dynamics and heterodimensional cycles, Ergodic Theory Dynam. Systems 15 (1995) 291-315.

[42] L.J. Díaz, E. Pujals, R. Ures, Partial hyperbolicity and robust transitivity, Acta Math. 183 (1999) 1-43.

[43] L.J. Díaz, J. Rocha, Large measure of hyperbolic dynamics when unfolding heterodimensional cycles, Nonlinearity 10 (1997) 857-884.

[44] D. Dolgopyat, On differentiability of SRB states for partially hyperbolic systems, Invent. Math. 155 (2004) 359-449.

[45] M. Feigenbaum, Qualitative universality for a class of nonlinear transformations, J. Statist. Phys. 19 (1978) $25-52$.

[46] J. Franks, R. Williams, Anomalous Anosov Flows. Global Theory of Dynamical Systems, Lecture Notes in Math., vol. 819, Springer, 1980.

[47] S.V. Gonchenko, L.P. Shil'nikov, D.V. Turaev, Dynamical phenomena in systems with structurally unstable Poincaré homoclinic orbits, Chaos 6 (1996) 15-31.

[48] A. Gorodetski, V. Kaloshin, How often surface diffeomorphisms have infinitely many sinks and hyperbolicity of periodic points near a homoclinic tangency, in press.

[49] J. Graczyk, G. Swiatek, Generic hyperbolicity in the logistic family, Ann. of Math. 146 (1997) 1-52.

[50] J. Guckenheimer, R.F. Williams, Structural stability of Lorenz attractors, Publ. Math. IHES 50 (1979) 59-72.

[51] S. Hayashi, Connecting invariant manifolds and the solution of the $C^{1}$ stability and $\Omega$-stability conjectures for flows, Ann. of Math. 145 (1997) 81-137.

[52] M. Hénon, A two dimensional mapping with a strange attractor, Comm. Math. Phys. 50 (1976) 69-77.

[53] Yu. Ilyashenko, W. Li, Nonlocal Bifurcations, Math. Surveys and Monographs, vol. 66, Amer. Math. Soc., 1999.

[54] M. Jakobson, On smooth mappings of the circle into itself, Math. USSR-Sb. 14 (1971) 161-185.

[55] M. Jakobson, Absolutely continuous invariant measures for one-parameter families of one-dimensional maps, Comm. Math. Phys. 81 (1981) 39-88.

[56] V.Yu. Kaloshin, B.R. Hunt, Stretched exponential estimate on growth of the number of periodic points for prevalent diffeomorphisms, in press.

[57] Yu. Kifer, Ergodic Theory of Random Perturbations, Birkhäuser, 1986.

[58] Yu. Kifer, Random Perturbations of Dynamical Systems, Birkhäuser, 1988.

[59] O. Kozlovski, Getting rid of the negative Schwarzian derivative condition, Ann. of Math. 152 (2000) 743-762.

[60] O. Kozlovski, Axiom A maps are dense in the space of unimodal maps in the $C^{k}$ topology, Ann. of Math. 157 (2003) 1-43.

[61] O. Kozlovski, W. Shen, S. van Strien, Density of hyperbolicity in dimension one, Preprint, Warwick, 2004.

[62] O. Kozlovski, S. van Strien, W. Shen, Rigidity for real polynomials, Preprint, Warwick, 2003.

[63] R. Labarca, M.J. Pacifico, Stability of singular horseshoes, Topology 25 (1986) 337-352.

[64] M. Levi, Qualitative analysis of the periodically forced relaxation oscillations, Mem. Amer. Math. Soc. 32 (244) (1981).

[65] N. Levinson, A second order differential equations with singular solutions, Ann. of Math. 50 (1949) 127-153.

[66] S.-T. Liao, Hyperbolicity properties of the non-wandering sets of certain 3-dimensional systems, Acta Math. Sci. 3 (1983) $361-368$.

[67] S.T. Liao, On the stability conjecture, Chinese Ann. of Math. 1 (1980) 9-30. 
[68] J. Littlewood, On non-linear differential equations of the second order, III, Acta Math. 97 (1957) 267-308.

[69] J. Littlewood, On non-linear differential equations of the second order, IV, Acta Math. 98 (1957) 1-110.

[70] E.N. Lorenz, Deterministic nonperiodic flow, J. Atmosph. Sci. 20 (1963) 130-141.

[71] M. Lyubich, Almost every real quadratic map is either regular or stochastic, Ann. of Math. 156 (2002) 1-78.

[72] R. Mañé, An ergodic closing lemma, Ann. of Math. 116 (1982) 503-540.

[73] R. Mañé, A proof of the $C^{1}$ stability conjecture, Publ. Math. IHES 66 (1988) 161-210.

[74] M. Martens, T. Nowicki, Invariant measures for Lebesgue typical quadratic maps. Géométrie complexe et systèmes dynamiques (Orsay, 1995), Astérisque 261 (2001) 239-252.

[75] R.M. May, Simple mathematical models with very complicated dynamics, Nature 261 (1976) 459-467.

[76] C. McMullen, Complex Dynamics and Renormalization, Ann. of Math. Stud., vol. 142, Princeton University Press, 1994.

[77] R. Metzger, Sinai-Ruelle-Bowen measures for contracting Lorenz maps and flows, Ann. Inst. H. Poincaré Anal. Non Linéaire 17 (2000) 247-276.

[78] R. Metzger, Stochastic stability for contracting Lorenz maps and flows, Comm. Math. Phys. 212 (2000) $277-296$.

[79] L. Mora, M. Viana, Abundance of strange attractors, Acta Math. 171 (1993) 1-71.

[80] C. Morales, M.J. Pacifico, A dichotomy for three-dimensional vector fields, Ergodic Theory Dynam. Systems 23 (2003) 1575-1600.

[81] C. Morales, M.J. Pacifico, E. Pujals, Singular hyperbolic systems, Proc. Amer. Math. Soc. 127 (1999) 3393-3401.

[82] C. Morales, M.J. Pacifico, E. Pujals, Robust transitive singular sets for 3-flows are partially hyperbolic attractors and repellers, Ann. of Math. 160 (2004) 1-58.

[83] C.G. Moreira, J. Palis, M. Viana, Homoclinic tangencies and fractal invariants in arbitrary dimension, C. R. Acad. Sci. Paris, Sér. I Math. 333 (2001) 475-480.

[84] C.G. Moreira, J.-C. Yoccoz, Stable intersections of regular Cantor sets with large Hausdorff dimensions, Ann. of Math. 154 (2001) 45-96; Corrigendum in: C.G. Moreira, J.-C. Yoccoz, Ann. of Math. 154 (2001) 527.

[85] S. Newhouse, Diffeomorphisms with infinitely many sinks, Topology 13 (1974) 9-18.

[86] S. Newhouse, The abundance of wild hyperbolic sets and nonsmooth stable sets for diffeomorphisms, Publ. Math. IHES 50 (1979) $101-151$.

[87] S. Newhouse, J. Palis, Cycles and bifurcation theory, Astérisque 31 (1976) 44-140.

[88] S. Newhouse, J. Palis, F. Takens, Bifurcations and stability of families of diffeomorphisms, Publ. Math. IHES 57 (1983) 5-71.

[89] J. Palis, On Morse-Smale dynamical systems, Topology 8 (1969) 385-405.

[90] J. Palis, A global view of Dynamics and a conjecture on the denseness of finitude of attractors. Géométrie complexe et systèmes dynamiques (Orsay, 1995), Astérisque 261 (1995) 335-347.

[91] J. Palis, S. Smale, Structural stability theorems, in: Global Analysis, Berkeley 1968, in: Proc. Sympos. Pure Math., vol. XIV, Amer. Math. Soc., 1970, pp. 223-232.

[92] J. Palis, F. Takens, Stability of parametrized families of gradient vector fields, Ann. of Math. 118 (1983) 383-421.

[93] J. Palis, F. Takens, Cycles and measure of bifurcation sets for two-dimensional diffeomorphisms, Invent. Math. 82 (1985) $397-422$.

[94] J. Palis, F. Takens, Hyperbolic and the creation of homoclinic orbits, Ann. of Math. 1987 (1987) 337-374.

[95] J. Palis, F. Takens, Hyperbolicity and Sensitive-Chaotic Dynamics at Homoclinic Bifurcations, Cambridge University Press, 1993.

[96] J. Palis, M. Viana, High dimension diffeomorphisms displaying infinitely many periodic attractors, Ann. of Math. 140 (1994) $207-250$.

[97] J. Palis, J.-C. Yoccoz, Homoclinic tangencies for hyperbolic sets of large Hausdorff dimension, Acta Math. 172 (1994) 91-136.

[98] J. Palis, J.-C. Yoccoz, Fers à cheval non uniformément hyperboliques engendrés par une bifurcation homocline et densité nulle des attracteurs, C. R. Acad. Sci. Paris, Sér. I Math. 333 (2001) 867-871.

[99] M. Peixoto, Structural stability on two-dimensional manifolds, Topology 1 (1962) 101-120.

[100] Ya. Pesin, Ya. Sinai, Gibbs measures for partially hyperbolic attractors, Ergodic Theory Dynam. Systems 2 (1982) 417-438.

[101] E. Pujals, Density of hyperbolicity and homoclinic bifurcation for 3d-diffeomorphisms in attracting regions, IMPA's preprint server, http://www.preprint.impa.br.

[102] E. Pujals, M. Sambarino, Homoclinic tangencies and hyperbolicity for surface diffeomorphisms, Ann. of Math. 151 (2000) $961-1023$.

[103] J. Robbin, A structural stability theorem, Ann. of Math. 94 (1971) 447-493.

[104] C. Robinson, Structural stability of vector fields, Ann. of Math. 99 (1974) 154-175; Errata in: C. Robinson, Ann. of Math. 101 (1975) 368.

[105] C. Robinson, Homoclinic bifurcation to a transitive attractor of Lorenz type, Nonlinearity 2 (1989) 495-518.

[106] A. Rovella, The dynamics of perturbations of the contracting Lorenz attractor, Bull. Braz. Math. Soc. 24 (1993) $233-259$.

[107] D. Ruelle, A measure associated with Axiom A attractors, Amer. J. Math. 98 (1976) 619-654.

[108] M. Rychlik, Lorenz attractors through Shil'nikov-type bifurcation. Part 1, Ergodic Theory Dynam. Systems 10 (1990) $793-821$.

[109] A. Sannami, The stability theorems for discrete dynamical systems on two-dimensional manifolds, Nagoya Math. J. 90 (1983) 1-55.

[110] L.P. Shil'nikov, On the generation of periodic motion from a trajectory doubly asymptotic to an equilibrium state of saddle type, Math. USSR-Sb. 6 (1968) 428-438.

[111] R. Simon, A 3-dimensional Abraham-Smale example, Proc. Amer. Math. Soc. 34 (1972) 629-630.

[112] Ya. Sinai, Gibbs measures in ergodic theory, Russian Math. Surveys 27 (1972) 21-69. 
[113] S. Smale, Diffeomorphisms with many periodic points, in: Differential and Combinatorial Topology, Princeton University Press, 1965.

[114] S. Smale, Differentiable dynamical systems, Bull. Amer. Math. Soc. 73 (1967) 747-817.

[115] D. Sullivan, Bounds, quadratic differentials and renormalization conjectures, Ann. Math. Soc. Centennial Publ. 2 (1992) $417-466$.

[116] L. Tedeschini-Lalli, J. Yorke, How often do simple dynamical systems have infinitely many coexisting sinks? Comm. Math. Phys. 106 (1986) 635-657.

[117] M. Tsujii, Physical measures for partially hyperbolic surface endomorphisms, Acta Math., in press.

[118] W. Tucker, A rigorous ODE solver and Smale's 14th problem, Found. Comput. Math. 2 (2002) 53-117.

[119] R. Ures, Abundance of hyperbolicity in $C^{1}$ topology, Ann. Sci. École Norm. Sup. 28 (1995) 747-760.

[120] C. Vasquez, Statistical stability for diffeomorphisms with dominated splitting, in press.

[121] M. Viana, Strange attractors in higher dimensions, Bull. Braz. Math. Soc. 24 (1993) 13-62.

[122] M. Viana, Multidimensional nonhyperbolic attractors, Publ. Math. IHES 85 (1997) 63-96.

[123] M. Viana, What's new on Lorenz strange attractors? Math. Intelligencer 22 (2000) 6-19.

[124] L. Wen, Homoclinic tangencies and dominated splittings, Nonlinearity 15 (2002) 1445-1469.

[125] L. Wen, Generic diffeomorphisms away from homoclinic tangencies and heterodimensional cycles, Bull. Braz. Math. Soc. 35 (2004) 419-452.

[126] L.-S. Young, Stochastic stability of hyperbolic attractors, Ergodic Theory Dynam. Systems 6 (1986) 311-319. 